Research Article

\title{
5G Edge Computing Enabled Directional Data Collection for Medical Community Electronic Health Records
}

\author{
Xiaoqiang Yan $\left.{ }^{1}\right)^{1}$ and Xiaogang Ren $\mathbb{i D}^{1,2}$ \\ ${ }^{1}$ The Affiliated Changshu Hospital of Soochow University (Changshu No. 1 People's Hospital), Jiangsu, 215500 Changshu, \\ Suzhou, China \\ ${ }^{2}$ China University of Mining Technology, Jiangsu, 221116 Xuzhou, China
}

Correspondence should be addressed to Xiaogang Ren; 1b20060005@cumt.edu.cn

Received 2 March 2021; Revised 13 May 2021; Accepted 9 July 2021; Published 23 July 2021

Academic Editor: Khin wee Lai

Copyright (c) 2021 Xiaoqiang Yan and Xiaogang Ren. This is an open access article distributed under the Creative Commons Attribution License, which permits unrestricted use, distribution, and reproduction in any medium, provided the original work is properly cited.

\begin{abstract}
It is important to promote the development and application of hospital information system, community health service system, etc. However, it is difficult to realize the intercommunication between various information systems because it is not enough to realize the in-depth management of health information. To address these issues, we design the 5G edge computing-assisted architecture for medical community. Then, we formulate the directional data collection (DDC) problem to gather the EMR/HER data from the medical community to minimize the service error under the deadline constraint of data collection deadline. Moreover, we design the data direction prediction algorithm (DDPA) to predict the data collection direction and propose the data collection planning algorithm (DCPA) to minimize the data collecting time cost. Through the numerical simulation experiments, we demonstrate that our proposed algorithms can decrease the total time cost by $62.48 \%$ and improve the data quality by $36.47 \%$ through the designed system, respectively.
\end{abstract}

\section{Introduction}

Recently, the core role of smart medicine is the construction of hospital information platform based on electronic medical records and regional health information platform with resident's electronic health records [1]. Electronic health records [2] can improve the phenomenon of information asymmetry between doctors and patients and satisfy the demand-oriented development of medical service reform. The construction of electronic health records is the focus of future smart medicine [3], which can not only satisfy the diversity requirements of medical and health reform but also accelerate and strengthen the development of information technology in medical and health institutions. Recently, the vigorous development of information technology in China's medical and health industry has given birth to the vigorous development and application of hospital information system (HIS), community health service system (CHSS), and other information systems. However, it is difficult to realize the intercommunication between various information systems because it is not enough to realize the in-depth management of health information.

The National Health Commission of the People's Republic of China [4] is committed to promoting the 5Gassisted medical action project. On the basis of medical and health information, it can promote not only the implementation of hierarchical diagnosis and treatment but also the reform of public medical institutions and public health management mode. Then, 5G-assisted medical care aims to improve the efficiency of medical institutions and integrate high-quality medical resources such as electronic health records (EHRs) [5], overcoming the shortcoming of traditional medical services and enabling patients and improving the upgrading of traditional medical service mode.

The scientific and technological application of medical community [6] electronic health records have been attracting the attention of many researchers and enterprises. Reference [7] compared achievement of and improvement 
in quality standards for diabetes at practices using EHRs with those at practices using paper records. Reference [7] examined the effects of electronic health records on the safety of patients in medical facilities. Reference [8] analyzed the costs and benefits of EHRs in six community health centers (CHCs) that serve disadvantaged patients.

However, the above researches do not deeply study the requirements and data characteristics of medical community platform for EHR management. In terms of credibility, reliability, and real-time, it is necessary to deeply study the directional collection mechanism of archival data, such as large-scale mobile terminal sensing under 5G, diversified archival data collection, and archival information sharing under the medical community.

So, there are some major challenges as follows:

(i) The diversity and complexity of medical community seriously restrict the classification efficiency and marking accuracy of medical community electronic health records data, which affects the intelligent management efficiency of EHRs.

(ii) The differences of medical level and service objects between different medical institutions in the medical community make the sharing of electronic records, which is an important basis for specialist collaboration, more complex. The low efficient and precision data sharing will seriously restrict the medical community's ability to solve major diseases, and it is difficult to form a complementary development mode.

(iii) How to accurately and timely collect the data of medical community electronic health record management has become a difficult problem because it is difficult to predict which medical structure will produce what type of electronic health record data at what time.

Our key contributions can be summarized as follows:

(i) We design the 5G edge computing-assisted architecture for medical community.

(ii) We formulate the directional data collection (DDC) problem to gather the EMR/HER data from the medical community with minimizing the service error under the deadline constraint of data collection.

(iii) We design the data direction prediction algorithm (DDPA) to predict the data collection direction and propose the data collection planning algorithm (DCPA) to minimize the data collecting time cost.

(iv) We conduct extensive simulations for the designed system and proposed algorithms. The results show that our proposed algorithms can decrease the total time cost by $62.48 \%$ and improve the data quality by $69.95 \%$ through the designed system, respectively.

The rest of the paper is organized as follows. We review the state-of-the-art research in Section 2. We design the 5Gassisted edge computing system in Section 3. We present the system model and formulate the DDC problem in Section 4. We design the intelligent data collection scheme with the assistance of random forest for solving the DDC problem in Section 5. We conduct the simulations in Section 6. We conclude this work in Section 7.

\section{Related Work}

The current situation of medical archives management in the medical community is discussed as follows. Reference [9] found the significant deficiencies in the practice of warfarin management and suggestive evidence that anticoagulation services can partially ameliorate these deficiencies. Reference [10] described a randomized trial of a program to identify and treat depression among high utilizers of general medical care. Reference [11] designed an intelligent archive management system by integrating 5G network and Internet of Things for smart hospitals. Reference [12] used the exome sequencing for infants in intensive care units to determine the diagnostic yield and use of clinical exome sequencing in critically ill infants. Reference [13] proposed a novel drug supply chain management using hyper ledger fabric based on block-chain technology to handle secure drug supply chain records.

About the status of data collection, reference [14] extracted security data that plays an important role in detecting security anomaly toward security measurement. Reference [15] provided a theoretical model of privacy in which data collection requires the consent of consumers who are fully aware of the consequences of consent. Reference [16] considered a scenario where an unmanned aerial vehicle collects data from a set of sensors on a straight line. Reference [17] proposed a low redundancy data collection scheme to reduce the delay as well as energy consumption for monitoring network by using matrix completion technique. Reference [18] proposed a practical framework called Privacy Protector, patient privacy-protected data collection, with the objective of preventing these types of attacks.

\section{5G-Assisted Edge Computing System}

First of all, according to the requirements of medical community e-health records management, based on the complex environment of regional medical institutions information platform, we design the mathematical model of e-health records management and its $5 \mathrm{G}$ application system framework. Secondly, by deploying multiple mobile terminal nodes, we design the medical community electronic health records management $5 \mathrm{G}$ architecture, to provide realtime and reliable communication guarantee for large-scale medical community electronic health records data application business. Then, in order to ensure the real-time and reliability of data sharing of medical community electronic health records, a massive data collection mechanism based on edge computing is established. Finally, based on the above requirements, we combine the large-scale mobile communication of $5 \mathrm{G}$ with the massive data real-time collection technology of edge computing to study the ap- 
plication mechanism of data directional collection, so as to provide the reliability, credibility, and feasibility guarantee for the data update and sharing application of medical community electronic health records management.

According to the requirements of regional medical institutions information platform construction, we analyze the information interconnection and regional differences between community health service centers and municipal hospitals. Then, we introduce edge computing into 5G through the organic allocation and deep integration between the mobile terminals and cloud computing server. The edge computing reasonably allocates the storage, computing, and network services resources between the computing center and the mobile terminals, so as to achieve the local optimal division of labor and cooperation before the network service quality and user experience quality. Therefore, the introduction of edge computing into $5 \mathrm{G}$ can satisfy the computing and communication needs of mobile terminals with distributed and random characteristics. Hence, edge computing can well solve the geographical deployment characteristics of $5 \mathrm{G}$ nodes scattered between community health service centers and municipal hospitals. Moreover, the edge computing architecture with $5 \mathrm{G}$ is shown in Figure 1. Here, the $5 \mathrm{G}$ platform is the center, i.e., the municipal hospital, and several subnets of edge community service center are deployed. The network control ability of these center subnets is the same as that of the servers in the platform, where the architecture can effectively reduce the calculation delay and improve the storage efficiency of medical community electronic health record data.

The 5G architecture shown in Figure 1 can provide convenient services, health management services, traditional Chinese medicine (TCM) health care services, and other services. This architecture can give full play to its advantages in data sharing and family doctor follow-up, continuously improve the accessibility and effectiveness of services, comprehensively improve service level and satisfaction, and provide medical services and health management services to the majority of residents conveniently and quickly. At the same time, the medical community platform can solve the following problem, lack of medical resources shortage, difficulty to see a doctor, and realize the integration of health resources and then improve the level of primary health care through the establishment of complete electronic health records for residents. Then, we integrate the sharing of medical records and test results, medical images, medication records, and patients' basic health information between secondary and tertiary comprehensive medical institutions in the community to realize the sharing of high-quality medical resources in the region.

With the rapid development of $5 \mathrm{G}$ edge terminals used to collect electronic health records data, how to reasonably allocate and effectively recover the diversity resources of $5 \mathrm{G}$ has become a key problem. In 5G environment, the distribution and recovery of resources and the reconstruction of network topology are dynamic. There is an unknown mapping and interference relationship between $5 \mathrm{G}$ real-time resource statistics, computing task resource allocation and task scheduling, and $5 \mathrm{G}$ network edge computing terminal trusted resource information. These relationships are realtime and random. It is the main goal of network resource management to make $5 \mathrm{G}$ system execution efficiency and resource utilization always in the best state. It is well known that $5 \mathrm{G}$ supports a large amount of traffic. The resource request queue is very easy to overflow, which makes the arrival rate and processing efficiency of resource request signaling and computing task control signaling between the network control center and the edge terminal irregular, and the reliability of resource allocation and computing task unbalanced among different services. In order to improve the instantaneous resource management level of $5 \mathrm{G}$ and the utilization rate of global resources and make $5 \mathrm{G}$ better communication support for medical community electronic health records management, we design the $5 \mathrm{G}$ edge computing architecture as shown in Figure 2, where we deployed with multiple edge terminals, multiple autonomous base stations, and multiple autonomous control networks.

In Figure 2, edge computing terminals share EHR information and exchange unified standard data sources through regional platform interfaces of medical institutions. According to the edge computing architecture shown in Figure 2, medical institutions improve the interconnection architecture of regional health information platform and guide the electronic medical record system and electronic health record management system of medical institutions under their jurisdiction. In particular, electronic health records need to achieve unified data interface standards of medical institutions, medical insurance, community, and other related systems, so as to facilitate information sharing.

The common data element established in the $5 \mathrm{G}$ control center of medical community can efficiently improve the real-time sharing efficiency of electronic medical record (EMR) and EHR. Therefore, the electronic medical records and electronic health records storage system of medical institutions in medical community must follow the standardized description of national public health data element attributes, describe the extracted data element attributes, conduct business modeling, and realize data sharing. Medical institutions at all levels should use the ID number as the main identification code for the transmission and circulation of information in the diagnosis and treatment of public health services in the business system, so as to ensure the effective collection of archival information. Each edge computing terminal server should ensure the validity and timeliness of data transmission, verification, process tracking, and traceability, so as to ensure that all kinds of information can be uploaded to $5 \mathrm{G}$ information platform timely, accurately, and comprehensively.

Therefore, how to collect and improve data from the community and medical institutions at all levels in accordance with the electronic medical record information standards and unified specification of disease coding and other important databases, to ensure the quality of data, has become the key of medical community archives management. The process of data collection should have the following properties: 


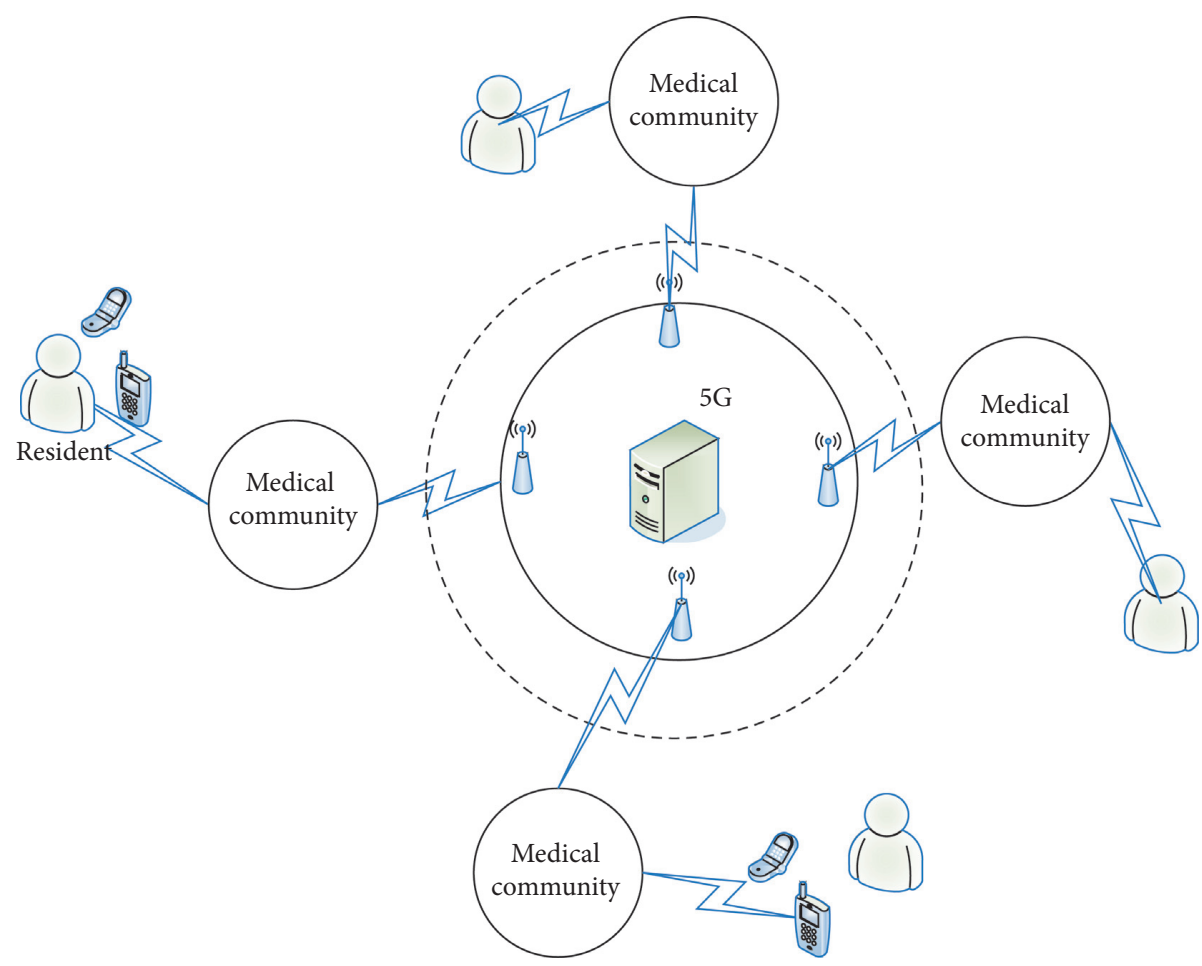

FiguRe 1: 5G architecture for medical community.

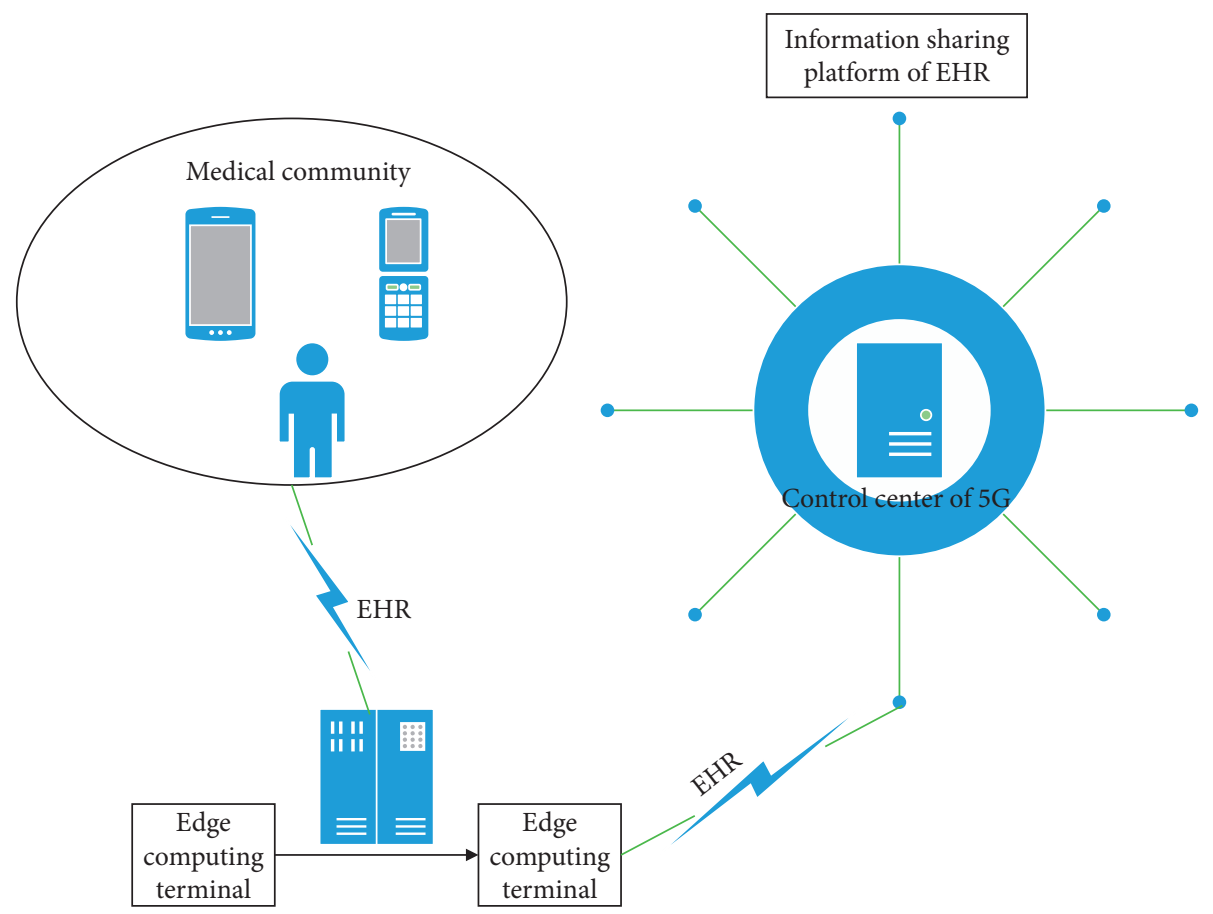

FIGURE 2: $5 \mathrm{G}$ edge computing architecture for medical community. 
(i) The process of data collection should be based on the main index of patient identity, correctly associated with the previous diagnosis and treatment data, and form a complete and standardized medical record file, which is convenient for medical staff to use.

(ii) The process of data collection should be carried out according to the interface specification of regional information platform. The diagnosis and treatment information should be uploaded and collected into health records in time.

(iii) The process of data collection should implement the codes of clinical symptoms, diagnosis, surgery, drugs, inspection, and so on released by the state to ensure the standardization and unification of diagnosis and treatment information uploaded to the platform.

Figure 3 gives a toy example for the Directional Data Collection and application of medical community electronic health record with the above characteristics. In this scenario, cloud platform, edge computing terminal, and 5G platform are effectively integrated into the directional data collection of medical community electronic health records, such as community classification, data directional collection, and data storage.

The edge computing procedure is illustrated as follows:

(1) The communities generate their EMR/HER data, which can be collected by a corresponding opportunistically encountered edge computing terminal.

(2) The edge computing terminal analyzes and mines the valid information from collected EMR/HER data, e.g., the location, resident ID, and his/her historical records. Then, the dataset is sent to the cloud servers in real time. The edge computing terminal predicts their medical demand according to the historical records by using the algorithm proposed in Section 4.1 and returns the prediction results to the $5 \mathrm{G}$ cloud servers.

(3) The 5G cloud servers periodically compute the data collection route via the algorithms in Section 4.2 for data collection terminal according to the information of communities and residents from edge computing terminals.

(4) The data is collected by the data collection terminal through the route calculated in the above step.

\section{Directional Data Collection for Medical Community}

4.1. System Model. Suppose that the $5 \mathrm{G}$ platform of medical community includes $m$ medical institutions and $n$ communities. According to the scope of service and medical level, the mapping relationship between each community and different medical institutions is established, denoted by $C=\left\{c_{i j}\right\}_{i=1, j=1}^{m, n}$. Here, the electronic file data is generated by the medical institutions according to the community it

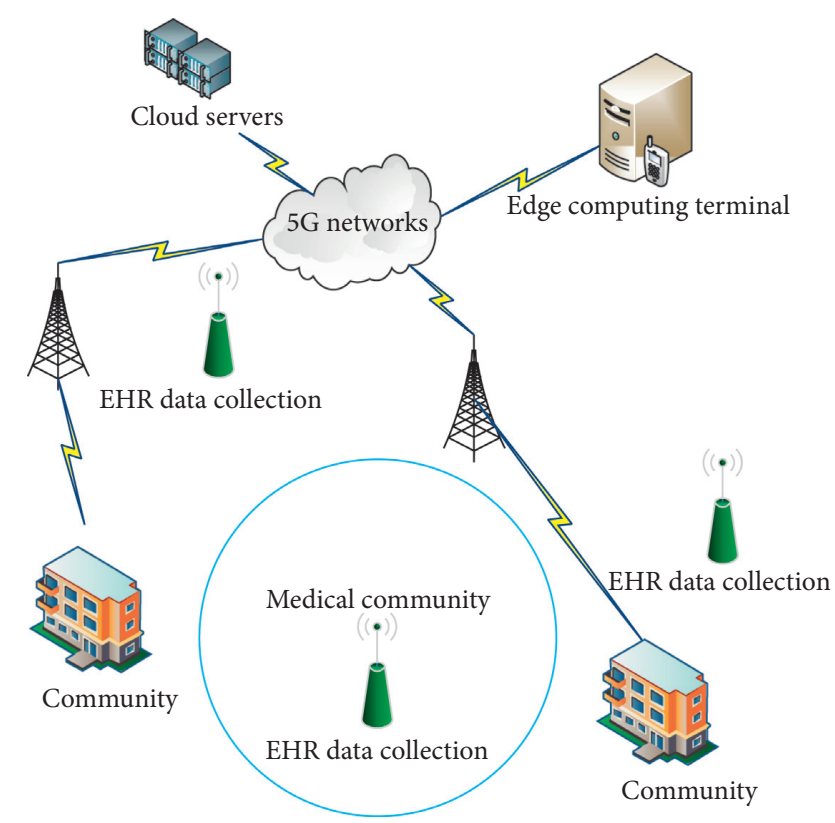

FIgURE 3: Application of directional data collection with the assistance of $5 \mathrm{G}$ and edge computing system.

serves, which is used to obtain the medical needs and feedback of residents. Note that each medical institution initiates one data collection task in the time dimension. So, $T=\left\{t_{i}\right\}_{i=1}^{m}$ denotes the task set. For convenience, we define the data collection process as follows.

Definition 1 (data collection task). The process of edge computing terminal completing data collection task is to select the corresponding observation values from a series of candidate data samples corresponding to the community for $5 \mathrm{G}$ platform.

For each data collection task $t_{i}$, we assume that it has a candidate sample set $A_{i}$ shown as follows:

$$
A_{i}=\left\{a_{i}(1), a_{i}(2), \ldots, a_{i}\left(\left|A_{i}\right|\right)\right\} .
$$

Then, let $a_{i}(0)$ denote the optimal data sample corresponding to the task $t_{i}$. Therefore, the data error caused by task $t_{i}$ can be calculated by

$$
e_{i}=\frac{1}{\left|A_{i}\right|}\left(\sum_{k=1}^{\left|A_{i}\right|} a_{i}(k)-a_{i}(0)\right) .
$$

The resident set who provides the EMR/HER dataset from all communities can be represented as follows:

$$
B_{i}=\left\{b_{i}^{j}\right\}_{j=1}^{n} .
$$

Here, $b_{i}^{j}$ represents the data in task $t_{i}$, which is provided by the $j$-th community when the $i$-th task is initiated by $i$-th medical institution when there is the mapping relationship belonging to $C$. So, we can calculate the data matrix corresponding to data collection tasks from all communities through the following equation: 


$$
\begin{aligned}
A & =\left\{a_{i j}\right\}_{i=1, j=1}^{m, n} \\
& =\prod_{i=1}^{m}\left(A_{i}-e_{i} B_{i}\right) \\
& =\prod_{i=1}^{m}\left(\sum_{k=1}^{\left|A_{i}\right|} \int a_{i}(k)^{t} \mathrm{~d} t-e_{i} B_{i}\right) \\
& =\prod_{i=1}^{m}\left(\sum_{k=1}^{\left|A_{i}\right|} \int a_{i}(k)^{t} \mathrm{~d} t-e_{i} \sum_{j=1}^{n} b_{i}^{j}\right) .
\end{aligned}
$$

Here, we redefine the data sample $a_{i}(k)$ in $A_{i}$ as $a_{i}(k)^{t}$, which is calculated according to the time dependence of edge computing. Note that we can update the mapping relationship between each community and different medical institutions through the following equation:

$$
c_{i j}= \begin{cases}0, & \text { if } 0<\frac{a_{i j}}{e_{i}}<\gamma_{i j}, \\ 1, & \text { if } \frac{a_{i j}}{e_{i}} \geq \gamma_{i j} .\end{cases}
$$

Here, $\gamma_{i j}$ is the tolerance threshold of the data collection error, which is a given empirical value.

In order to analyze the direction accuracy of data collection conveniently, we give the following definition of data collection aggregation reliability.

Definition 2 (data collection task). For $m$ data collection tasks and $n$ communities, the direction of data collection is accurate when each task satisfies the following conditions:

(1) The $i$-th task and $j$-th community have the mapping relationship, i.e., $c_{i j}=1$.

(2) $A_{i}$ and $B_{i}$ have the same rank for each task $t_{i}$.

(3) All the data samples collected by all the medical institutions have consistency; i.e., the following function $f(A)$ is valid:

$$
f(A)=\lim _{n \longrightarrow \infty} \frac{\lim _{m \longrightarrow \infty} A}{\sum_{i^{\prime}=1}^{i} r_{i}^{\prime}+\sum_{e \in T_{i}} t_{e}}
$$

(4) Let $\Gamma\left(t_{i}\right)$ denote the data collection time cost. The time cost of the $i$-th task is not larger than the deadline $\tau_{i}$.

4.2. Problem Formulation. The objective of directional data collection (DDC) problem is to design a data collection scheme based on $5 \mathrm{G}$ edge computing system to gather the EMR/HER data from the medical community to minimize the service error under the deadline constraint of data collection. $x_{i j}$ is a binary variable to indicate whether data of task $t_{i}$ is collected from $j$-th community. $x_{i j}=1$ if data of task $t_{i}$ is collected from $j$-th community. $x_{i j}=0$ otherwise.

The DDC problem can be formulated as follows:

$$
\begin{array}{cll}
\text { DDC min } & x_{i j} \sum_{i=1}^{m} e_{i} & \\
& \text { (a) } x_{i j} \in\{0,1\}, & \forall t_{i} \in T ; \\
& \text { (b) } r\left(A_{i}\right)=r\left(B_{i}\right), & \forall t_{i} \in T ; \\
\text { s.t. } & \text { (c) } \prod_{i=1}^{m} \prod_{j=1}^{n} c_{i j}=1 ; & \\
& \text { (d) } \Gamma\left(t_{i}\right) \leq \tau_{i}, & \forall t_{i} \in T .
\end{array}
$$

Constraint (a) gives the value range of $x_{i, j}$. Constraint (b) ensures that the rank of candidate data sample set is equal to that of data in task $t_{i}$ on the basis of $C$. Constraint (c) ensures that each mapping between the task and its corresponding community is valid. Constraint (d) ensures that the time cost of data collection in each task is not larger than its deadline.

We list the frequently used notations in Table 1.

\section{Intelligent Data Collection Scheme}

In this section, we propose our 5G edge computing enabled directional data collection (5EDDC) algorithm. Then, the detailed description of the proposed algorithm is presented in two phases: data direction prediction and data collection planning.

5.1. Data Direction Prediction Algorithm. Medical behaviors and requirements of residents in different communities actually reflect the regeneration direction of EMR, which contains a lot of medical information, such as common diseases and medical habits. Based on the implementation of a variant of algorithms [19], we design Algorithm 1 for solving the problem of data direction prediction.

First, we predict the medical behavior of residents through the following steps:

Step 1. Feature extraction takes into account the following features for each behavior in community generating along the historical records $B_{i}$ with the last data collection task $t_{i}$ and candidate sample set $A_{i}$ of current medical community: time of day, medical behavior starting time, medical community name and its location, resident ID, and medical treatment time of each location. The above procedure is denoted as function Feature Extraction (dataset).

Step 2. Random forest-based prediction needs the input vector $n_{t}$ representing the information of a resident, which includes the origin community and destination medical institute, as well as the corresponding extracted features from step 1 . Then, the data generating time of any community can be predicted based on temporal 
TABLE 1: Frequently used notations.

\begin{tabular}{|c|c|}
\hline Notation & Description \\
\hline$c_{i j}$ & Mapping relationship between $i$-th community and $j$-th different medical institution \\
\hline$C$ & Set of mappings \\
\hline$t_{i}$ & $i$-th data collection task \\
\hline$T$ & Set of tasks \\
\hline$A_{i}$ & Set of candidate data samples belonging to $i$-th task \\
\hline$a_{i}(k)$ & $k$-th data sample in $A_{i}$ \\
\hline$\left|A_{i}\right|$ & Size of data sample in $A_{i}$ \\
\hline$a_{i}(0)$ & Optimal data sample corresponding to task $t_{i}$ \\
\hline$e_{i}$ & Data error caused by task $t_{i}$ \\
\hline$b_{i}^{j}$ & Data in task $t_{i}$ provided by the $j$-th community when the $i$-th task is initiated by $i$-th medical institution \\
\hline$B_{i}$ & Set of data in task $t_{i}$ on the basis of $C$ \\
\hline$a_{i}(k)^{t}$ & $k$-th data sample calculated according to the time dependence of edge computing \\
\hline$\gamma_{i j}$ & $\begin{array}{c}\text { Tolerance threshold of the data collection error between the } i \text {-th task and } j \text {-th community } \\
\text { Consistence function }\end{array}$ \\
\hline$\tau_{i}$ & Deadline of task $t_{i}$ \\
\hline
\end{tabular}

data dependencies and spatial data correlations. The above process is denoted as function Random Forests.

Second, we can predict the data direction of community for collecting data via the Interval-based Historical Average (IHA) [20] as shown in the following equation:

$$
\operatorname{IHA}\left(E\left[t_{a}\right], \lambda\right)=\frac{1}{2 \lambda+1} \sum_{p=-\lambda}^{\lambda}\left(\sum_{q=1}^{\text {days }}\left(t_{p}-E\left[t_{a}\right]-q\right)\right) \text {. }
$$

Here, $E\left[t_{a}\right]$ is the expectation of data generating time of task $t_{a}$ based on historical EMR/HER data, $\lambda$ is the mean absolute error of the random forests [21] for data generating time prediction, and days is the days of historical EMR/EHR dataset. $t_{p}$ is the first data collection starting time after $E\left[t_{a}\right]+q$ in the $p$-th day of the dataset, where $E\left[t_{a}\right]+q$ is the generating time of EMR/HER data for recording residents in $a$-th community at the $q$-th day. Thus, $t_{p}-E\left[t_{a}\right]-q$ is the historical data collection time on the $q$-th day. Finally, we calculate the EMR/EHR data generating time of communities and data collection direction of medical community.

In Algorithm 1, the $\operatorname{Sink}(F, B)$ function is used to find all the data collection sinks of EMR/EHR at its medical community. In addition, Algorithm 1 updates all the data samples and records between any two communities and computes their collecting time through the following steps:

(1) Find all $\bar{a}_{i}$, which can satisfy the tolerance

(2) Extract all the features from historical dataset

5.2. Data Collection Planning Algorithm. Based on the direction prediction of data collection, the DDC problem is equivalent to finding a data route to collect the EMR/HER data for all selected medical communities with minimum time cost. The above data collection planning (DCP) problem can be formulated as follows:

$$
\mathrm{DCP} \min \sum_{0=1}^{C} \Gamma\left(t_{o}\right)
$$

Moreover, we design the data collection planning algorithm (DCPA) to solve the DCP problem. The basic idea is given as follows (see Algorithm 2):

(1) Transform $A_{i}$ and integrate into $\widetilde{A}$ (line 5)

(2) Update $\widetilde{B}$ (line 6)

(3) For each element in $\widetilde{B}$, we first divide it into two separate sets $P_{1}$ and $P_{2}$, and then remove half of elements in $\widetilde{B}$ (lines $8-10$ ), and then find the corresponding subroutes (lines 11-14)

(4) Integrate all the subroutes into the final data collection planning $P$ (lines 15-18) where the symbol $\uplus$ represents the integration of some routes.

\section{Numerical Experiments}

In this section, we conduct extensive simulations to verify the performance of our proposed algorithms with different number of medical institutions, number of communities, days of month, and number of residents of a community.

6.1. Data Description. The dataset used in our experiments is from the electronic records system of Changshu No. 1 People's Hospital. The dataset shows a kind of representative medical community data. It is generated by the medical community supported by Changshu No. 1 People's Hospital, which covers the period from January 1, 2018, to November 30,2018 . The dataset includes the record data of medical institute and residents, and GPS data of medical institutes. The data record contains various fields, such as time of day, medical behavior starting time, medical community name and its location, resident ID, medical treatment time of each 
Input: $T$, dataset

Output: $A, B$

(1) for $i=2$ to $m$ do

(2) $e_{i} \longleftarrow\left(1 /\left|A_{i}\right|\right)\left(\sum_{k=1}^{\left|A_{i}\right|} a_{i}(k)-a_{i}(0)\right)$

(3) $\bar{A}_{i} \longleftarrow\left\{\bar{a}_{i} \mid \bar{a}_{i}<e_{i}\right\}$;

(4) for $j=1$ to $n$ do

(5) $b_{i}^{j} \longleftarrow \bar{a}_{i}(j)$;

(6) for each $a \in A$ do

(7) $F \longleftarrow$ Feature Extraction (dataset);

(8) $\bar{B} \longleftarrow \operatorname{Sink}(F, B)$;

(9) for each $\bar{b} \in \bar{B}$ do

(10) $\quad(A, B) \longleftarrow$ Random Forests $(F, \bar{A}, \bar{B})$;

Algorithm 1: Data direction prediction algorithm (DDPA).

$$
\begin{aligned}
& \text { Input: } A, B \\
& \text { Output: } P \\
& \text { (1) count } \longleftarrow 0 ; \widehat{A} \longleftarrow A ; \widetilde{B} \longleftarrow \varnothing \text {; } \\
& \text { (2) while count } \leq \log B-1 \text { do } \\
& \text { (3) for } i=1 \text { to } m \text { do } \\
& \text { (4) for } j=1 \text { to } n \text { do } \\
& \text { (5) } \quad \widetilde{A} \longleftarrow\left\{\tilde{a}_{i}(1), \tilde{a}_{i}(2), \ldots, \tilde{a}_{i}\left(\left|A_{i}\right|\right)\right\} \\
& \text { (6) } \quad \widetilde{B} \longleftarrow\left\{\widetilde{b}_{i}^{j}\right\} \cup \widetilde{B} \text {; } \\
& \text { (7) for each } \widetilde{b} \in \widetilde{B} \text { do } \\
& \text { (8) Take }(|\widetilde{B}| / 2) \text { elements to } P_{1} \text {; } \\
& \text { (9) Take another }(|\widetilde{B}| / 2) \text { elements to } P_{2} \text {; } \\
& \text { (10) Remove }(|\widetilde{B}| / 2) \text { elements from } \widetilde{B} \text {; } \\
& \text { (11) Find subroute } p_{1}^{\text {count }} \text { of } P_{1} \text {; } \\
& \text { (12) } P_{1}^{\text {count }} \longleftarrow P_{1}^{\text {count }} \cup\left\{p_{1}^{\text {count }}\right\} \text {; } \\
& \text { (13) Find subroute } p_{2}^{\text {count }} \text { of } P_{2} \text {; } \\
& \text { (14) } \quad P_{2}^{\text {count }} \longleftarrow P_{2}^{\text {count }} \cup\left\{p_{2}^{\text {count }}\right\} \text {; } \\
& \text { (15) for each } p_{1} \in P_{1} \text { do } \\
& \text { (16) } \quad P \longleftarrow P \uplus\left\{p_{1}\right\} \text {; } \\
& \text { (17) for each } p_{2} \in P_{2} \text { do } \\
& \text { (18) } \quad P \longleftarrow P \uplus\left\{p_{2}\right\} \text {; }
\end{aligned}
$$

Algorithm 2: Data Collection Planning Algorithm (DCPA).

location, and the number of patients of the corresponding medical institute, etc. The GPS data contains latitude, longitude, and treatment time of medical institute.

6.2. Simulation Setup and Benchmark. We assume that there are 10 medical institutions to provide medical care for 10 communities, which are supported by Changshu No. 1 People's Hospital. The residents receive medical treatment from the above medical institutions. In our simulation, we evaluate the total time cost of data collection, and data quality calculated by equation (2). All the simulations were run on a cloud server ECS [23] with 12-core Intel Xeon Platinum 8269CY and $48 \mathrm{~GB}$ memory. The other parameter settings of our simulations are listed in Table 2.

We develop the data collection algorithm DCA in [22] as the benchmark algorithm for comparison, which can make an efficient tradeoff between the data collection efficiency and energy consumption through the combination of the energy of the emotional device wireless device.

6.3. Performance Evaluation. In this subsection, we evaluate the performance of our algorithms and DCA in the scenario shown in Figure 4 . Tables 3 and 4 give the locations of medical institutions and communities in the area, respectively. The above information is calculated based on Google Maps.

Figure 5 shows the prediction errors on two different days, i.e., March 10, 2018, and October 20, 2018. The prediction results demonstrate the effectiveness of our proposed prediction algorithm DDPA. The average prediction error on March 10, 2018, and October 20, 2018, is 3.21\% and $1.93 \%$, respectively.

Figures 6 and 7 show the impact of medical institutions on total time cost and data quality of our algorithms and 


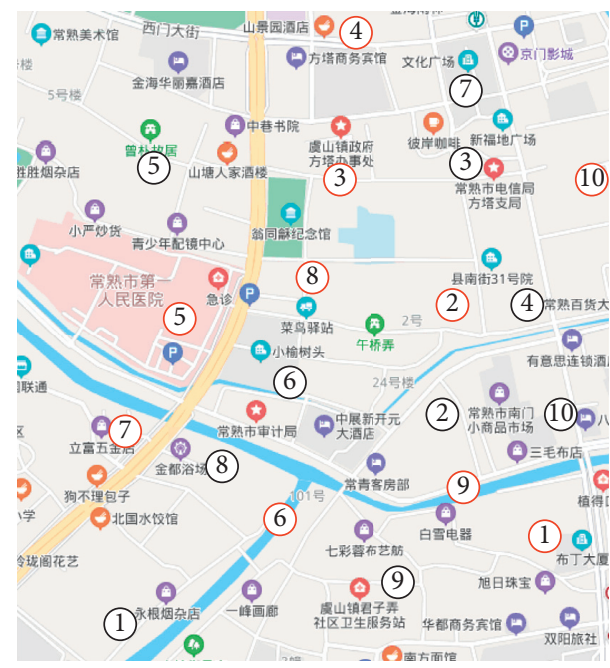

FIgUre 4: Medical community for EMR/HER data collection. The red nodes represent locations of medical institutions and the green nodes represent locations of communities.

TABle 2: Parameter settings.

\begin{tabular}{lc}
\hline Parameter & Value \\
\hline$n$ & 10 \\
$m$ & 10 \\
$|T|$ & 10 \\
$\gamma_{i j}$ & {$[0.01,0.05]$} \\
$\tau_{i} \mid$ & {$[1,10]$ hours } \\
$\left|A_{i}\right|$ & {$[100,500]$} \\
\hline
\end{tabular}

TABLE 3: Locations of medical institutions.

\begin{tabular}{lcc}
\hline$i$ & Longitude & Latitude \\
\hline 1 & 31.629934025445163 & 120.74572694707317 \\
2 & 31.637070592885376 & 120.74498723499269 \\
3 & 31.641308857555806 & 120.74260543350061 \\
4 & 31.644304755694158 & 120.743120417607 \\
5 & 31.639559503878488 & 120.73996684991539 \\
6 & 31.63350810894618 & 120.74108193885252 \\
7 & 31.634421579375328 & 120.73520253697116 \\
8 & 31.640051243952147 & 120.74334217244697 \\
9 & 31.63600474671818 & 120.74427558114591 \\
10 & 31.640862982947134 & 120.74781351639375 \\
\hline
\end{tabular}

TABLE 4: Locations of communities.

\begin{tabular}{lcc}
\hline$i$ & Longitude & Latitude \\
\hline 1 & 31.633639306384033 & 120.73640714235745 \\
2 & 31.635638094098336 & 120.74413897983557 \\
3 & 31.64162333734517 & 120.74648660051481 \\
4 & 31.638983794798012 & 120.74880870356253 \\
5 & 31.641820359193588 & 120.73842118763615 \\
6 & 31.63745217402931 & 120.74375621572658 \\
7 & 31.643893501251643 & 120.74744351115115 \\
8 & 31.63659402090503 & 120.742072053155 \\
9 & 31.634475760812066 & 120.74353931598635 \\
10 & 31.634834238968633 & 120.74956147326746 \\
\hline
\end{tabular}

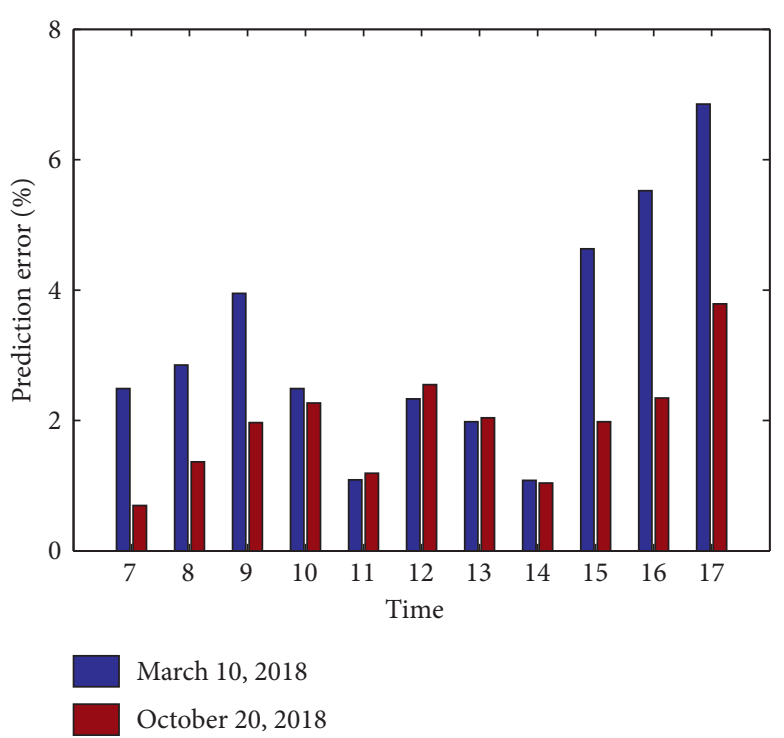

Figure 5: Prediction errors on March 10, 2018, and October 20, 2018.

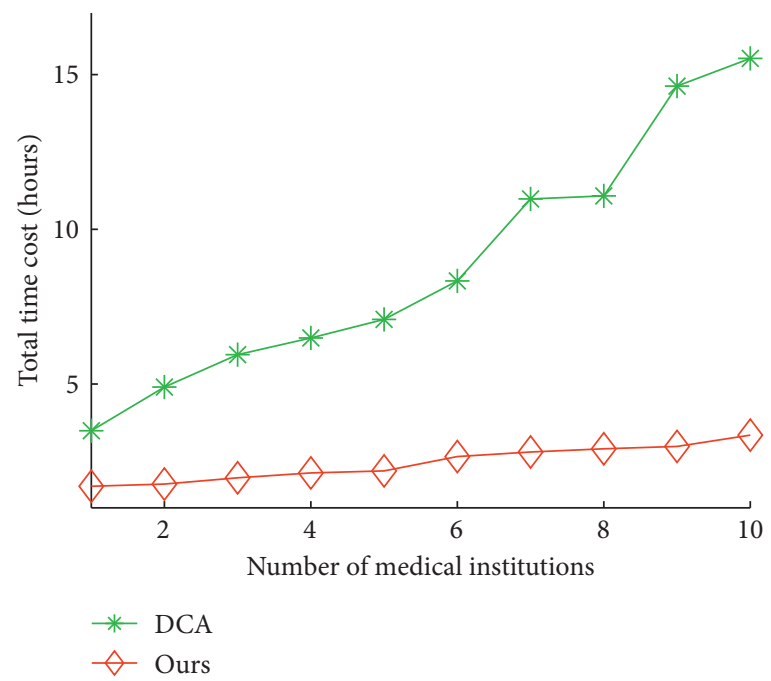

FIgURE 6: Total time cost vs. number of medical institutions.

DCA, respectively. The average total time cost of our algorithms and DCA is 8.85 hours and 2.44 hours, respectively. The average data quality of our algorithms and DCA is $75.76 \%$ and $94.75 \%$, respectively. The results show that our algorithms can reduce $72.38 \%$ of total time cost of DCA on average, and improve $25.06 \%$ of data quality of DCA, respectively. This indicates that the proposed algorithms significantly outperform DCA. This is because the data quality of our algorithms is better than the those obtained by DCA, respectively.

Figures 8 and 9 show the impact of communities on total time cost and data quality of our algorithms and DCA, respectively. The average total time cost of our algorithms and DCA is 5.75 hours and 1.63 hours, respectively. The average data quality of our algorithms and DCA is $73.27 \%$ and $97.59 \%$, respectively. The results show that our 


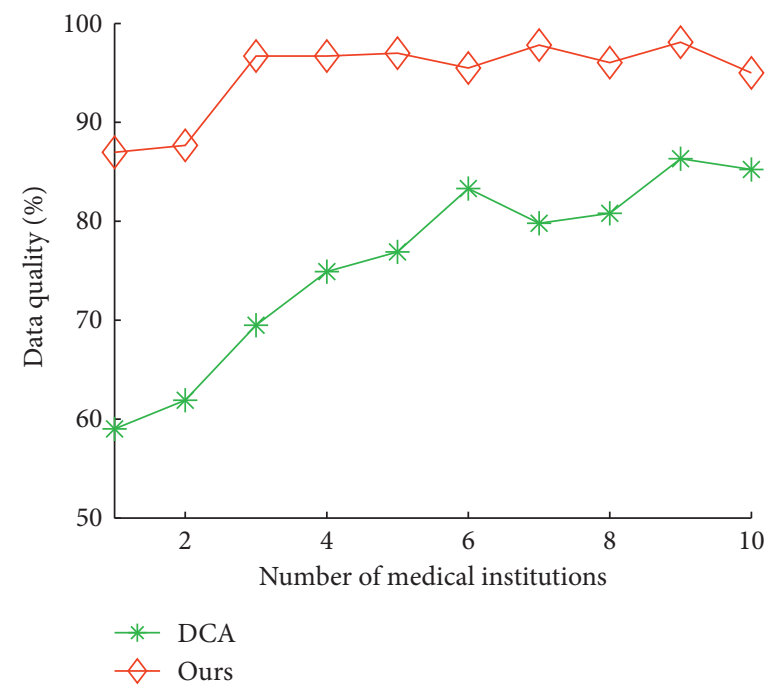

FIgURE 7: Data quality vs. number of medical institutions.

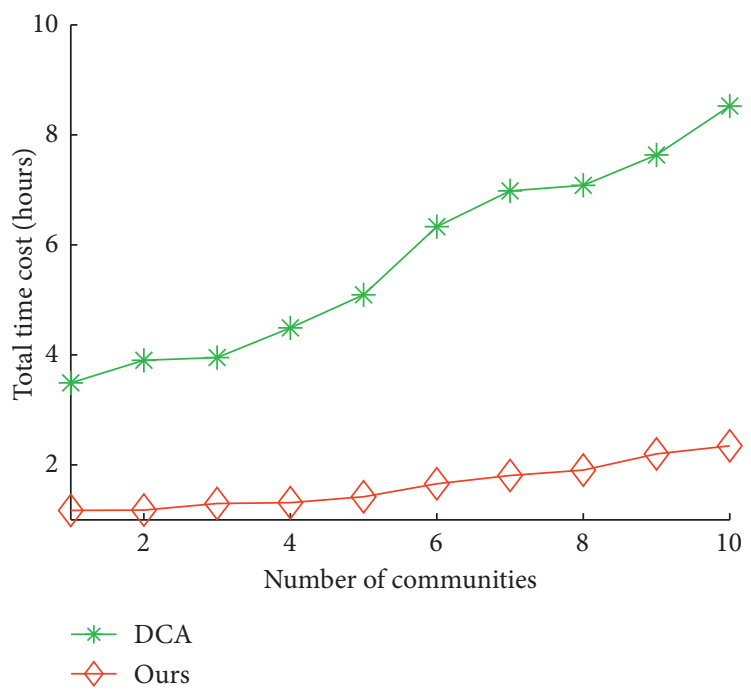

Figure 8: Total time cost vs. number of communities.

algorithms reduces $71.67 \%$ of total time cost of DCA on average, and improves $33.20 \%$ of data quality of DCA, respectively.

Figures 10 and 11 show the impact of days of month on total time cost and data quality of our algorithms and DCA, respectively. The average total time cost of our algorithms and DCA is 16.15 hours and 9.99 hours, respectively. The average data quality of our algorithms and DCA is $63.31 \%$ and $94.01 \%$, respectively. The results show that our algorithms can reduce $38.12 \%$ of total time cost of DCA on average, and improve $48.49 \%$ of data quality of DCA, respectively.

Figures 12 and 13 show the impact of communities on total time cost and data quality of our algorithms and DCA, respectively. The average total time cost of our algorithms and DCA is 8.92 hours and 2.86 hours, respectively. The average data quality of our algorithms and DCA is $68.44 \%$ and $95.21 \%$, respectively. The results show that our 


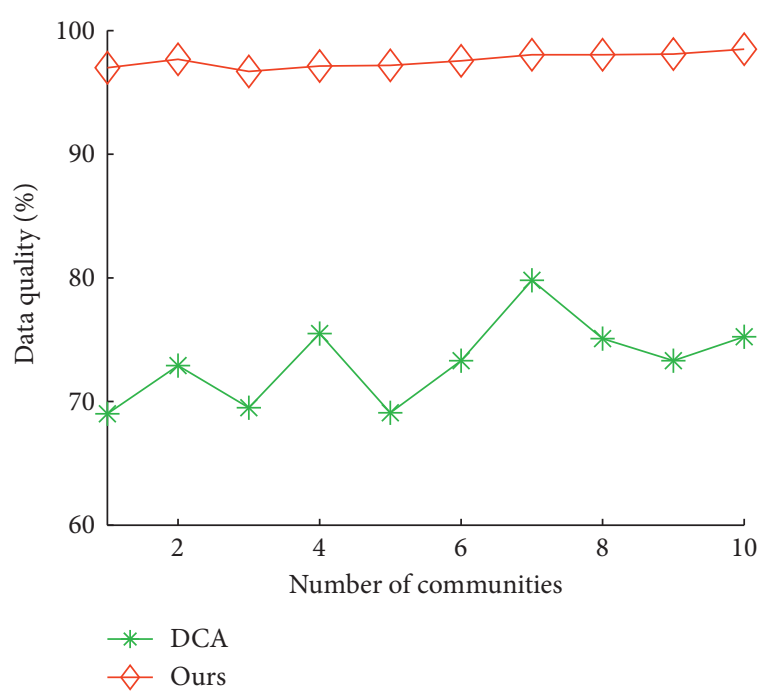

FIgURE 9: Data quality vs. number of communities.

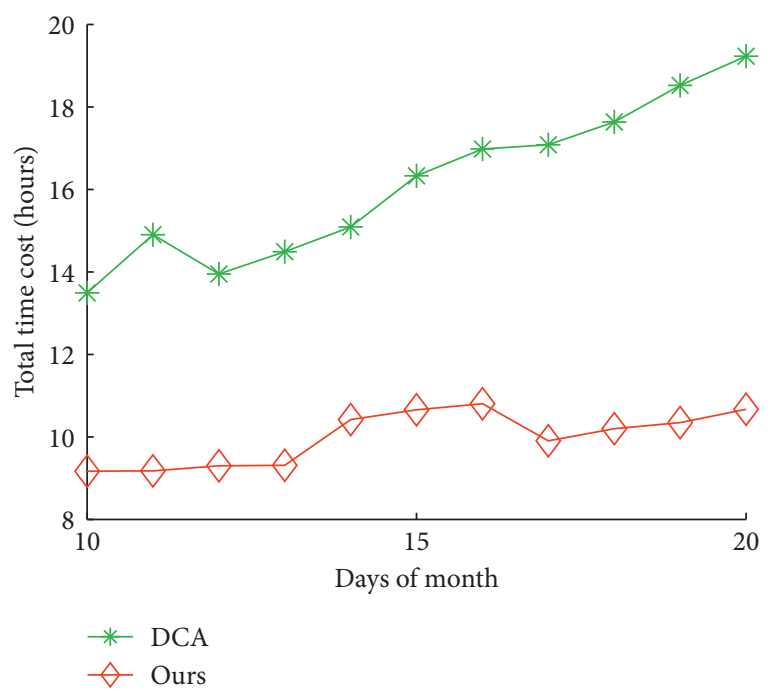

Figure 10: Total time cost vs. days of month.

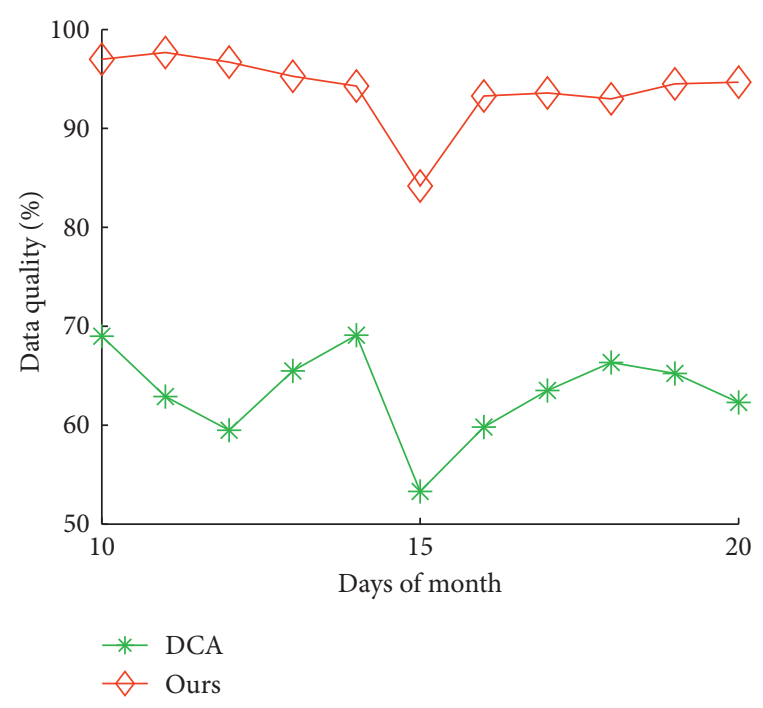

Figure 11: Data quality vs. days of month.

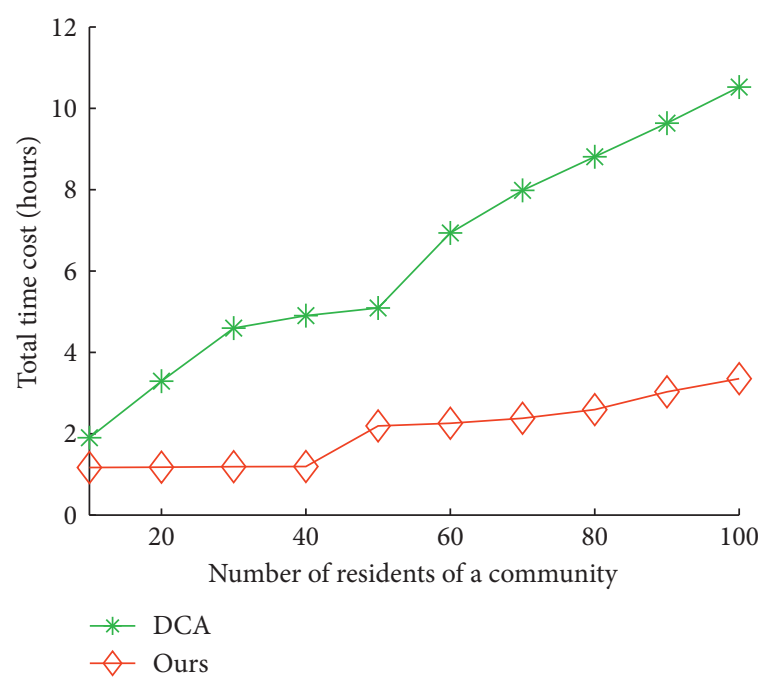

FIGURE 12: Total time cost vs. number of residents of a community.

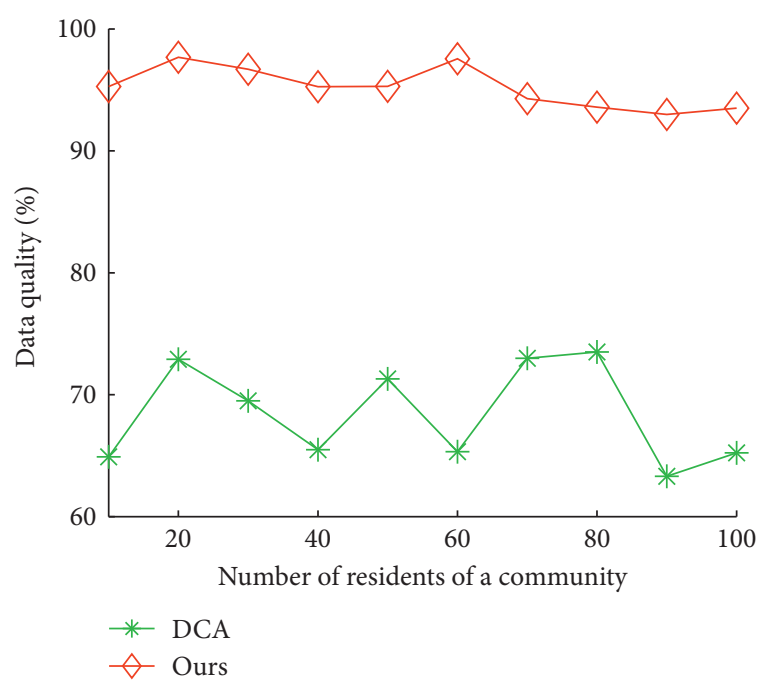

Figure 13: Data quality vs. number of residents of a community.

algorithms can reduce $67.76 \%$ of total time cost of DCA on average and improve $39.11 \%$ of data quality of DCA, respectively.

Overall, our algorithms can significantly decrease the total time cost and improve the data quality through the designed data direction prediction algorithm and data collection planning algorithm.

\section{Conclusion}

In this article, we have designed the 5G edge computing architecture for medical community to improve the effectiveness and efficiency of EMR/EHR data collection. First, we formulate the directional data collection (DDC) problem to gather the EMR/HER data from the medical community for minimizing the service error under the deadline constraint of data collection deadline. Second, we design the data direction prediction algorithm (DDPA) to predict the data collection direction, and propose the data collection 
planning algorithm (DCPA) to minimize the data collecting time cost. Finally, through the numerical simulation experiments, we demonstrate that our proposed algorithms can decrease the total time cost by $62.48 \%$ and improve the data quality by $36.47 \%$ through the designed system, respectively.

\section{Data Availability}

The labeled dataset used to support the findings of this study is available from the corresponding author upon request.

\section{Conflicts of Interest}

The authors declare no conflicts of interest.

\section{References}

[1] Z. Liu, C. Bai, H. Yu et al., “An adaptive deep learning model to differentiate syndromes of infectious fever in smart medicine," Future Generation Computer Systems, vol. 111, pp. $853-858,2020$.

[2] A. K. Jha, C. M. DesRoches, E. G. Campbell et al., "Use of electronic health records in U.S. hospitals," New England Journal of Medicine, vol. 360, no. 16, pp. 1628-1638, 2009.

[3] P. Elanthiraiyan and D. S. Babu, "Smart medicine and physical health system using IoT," International Journal of Computer Science and Mobile Computing, vol. 4, no. 3, pp. 333-338, 2015.

[4] National Health Commission of the People's Republic of China, http://www.nhc.gov.cn/.

[5] D. Blumenthal and M. Tavenner, "The "meaningful use" regulation for electronic health records," New England Journal of Medicine, vol. 363, no. 6, pp. 501-504, 2010.

[6] F. Mostashari, M. Tripathi, and M. Kendall, "A tale of two large community electronic health record extension projects," Health Affairs, vol. 28, no. 2, pp. 345-356, 2009.

[7] M. Z. Hydari, R. Telang, and W. M. Marella, "Electronic health records and patient safety," Communications of the $A C M$, vol. 58, no. 11, pp. 30-32, 2015.

[8] R. H. Miller and C. E. West, "The value of electronic health records in community health centers: policy implications," Health Affairs, vol. 26, no. 1, pp. 206-214, 2007.

[9] G. P. Samsa, D. B. Matchar, L. B. Goldstein et al., "Quality of anticoagulation management among patients with atrial fibrillation: results of a review of medical records from 2 communities," Archives of Internal Medicine, vol. 160, no. 7, pp. 967-973, 2000

[10] D. J. Katzelnick, G. E. Simon, S. D. Pearson et al., "Randomized trial of a depression management program in high utilizers of medical care," Archives of Family Medicine, vol. 9, no. 4 , pp. 345-351, 2000.

[11] F. Qiu, "Hospital archives intelligent management system based on 5G network and internet of things system," Microprocessors and Microsystems, vol. 80, Article ID 103564, 2021.

[12] L. Meng, M. Pammi, A. Saronwala et al., "Use of exome sequencing for infants in intensive care units," JAMA Pediatrics, vol. 171, no. 12, Article ID e173438, 2017.

[13] F. Jamil, L. Hang, K. Kim, and D. Kim, "A novel medical blockchain model for drug supply chain integrity management in a smart hospital," Electronics, vol. 8, no. 5, p. 505, 2019.
[14] H. Xie, Z. Yan, Z. Yao et al., "Data collection for security measurement in wireless sensor networks: a survey," IEEE Internet of Things Journal, vol. 6, no. 2, pp. 2205-2224, 2018.

[15] J. P. Choi, D.-S. Jeon, and B.-C. Kim, "Privacy and personal data collection with information externalities," Journal of Public Economics, vol. 173, pp. 113-124, 2019.

[16] J. Gong, T.-H. Chang, C. Shen, and X. Chen, "Flight time minimization of UAV for data collection over wireless sensor networks," IEEE Journal on Selected Areas in Communications, vol. 36, no. 9, pp. 1942-1954, 2018.

[17] J. Tan, W. Liu, M. Xie et al., “A low redundancy data collection scheme to maximize lifetime using matrix completion technique," EURASIP Journal on Wireless Communications and Networking, vol. 2019, no. 1, pp. 1-29, 2019.

[18] E. Luo, M. Z. A. Bhuiyan, G. Wang, M. A. Rahman, J. Wu, and M. Atiquzzaman, "PrivacyProtector: privacy-protected patient data collection in IoT-based healthcare systems," IEEE Communications Magazine, vol. 56, no. 2, pp. 163-168, 2018.

[19] Y. Wang, S. T. Xia, Q. Tang, J. Wu, and X. Zhu, "A novel consistent random forest framework: Bernoulli random forests," IEEE Transactions on Neural Networks and Learning Systems, vol. 29, no. 8, pp. 3510-3523, 2018.

[20] P. He, G. Jiang, S. Lam et al., "Travel-time prediction of bus journey with multiple bus trips," IEEE Transactions on Intelligent Transportation Systems, vol. 20, no. 11, pp. 41924205, 2019.

[21] L. Breiman, "Random forests," Machine Learning, vol. 45, no. 1, pp. 5-32, 2001

[22] “Aliyun ECS cloud server," 2021, https://www.aliyun.com/ product/ecs.

[23] Y. Jin, Z. Qian, and S. Chen, "Data collection scheme with minimum cost and location of emotional recognition edge devices," Personal and Ubiquitous Computing, vol. 23, no. 3-4, pp. 595-606, 2019. 